\title{
Self-Assembly of Bioconjugated Amphiphilic Mesogens Having Specific Binding Moieties at Aqueous-Liquid Crystal Interfaces
}

Hiroki Eimura, ${ }^{\dagger}$ Daniel S. Miller, ${ }^{\ddagger}$ Xiaoguang Wang, ${ }^{\ddagger}$ Nicholas L. Abbott, ${ }^{*, \ddagger}$ and Takashi Kato*, ${ }^{*}$

${ }^{\dagger}$ Department of Chemistry and Biotechnology, School of Engineering, The University of Tokyo, Hongo, Bunkyo-ku, Tokyo 113-8656, Japan

$\ddagger$ Department of Chemical and Biological Engineering, University of Wisconsin-Madison, 1415 Engineering Drive, Madison, Wisconsin 53706

\section{Supporting information}

Table of Contents:

1. Synthesis of compounds 1 and 2

2. Stability of monolayer for 2 on air-aqueous interface

3. Optical appearance under crossed polarizers of 5CB/1 and 5CB/2 between air and OTS-treated glass

4. Optical appearance under crossed polarizers of 5CB/1 between aqueous solution and OTS-

treated glass

5. References 


\section{Synthesis of compounds 1 and 2}

Compounds 1 and 2 were synthesized as shown in schemes S1 and S2. Compound 3, t-butyl 14hydroxy-3,6,9,12-tetraoxatetradecanoate, and protected RGD motif were prepared according to the method reported elsewhere. ${ }^{\text {S1-S3 }}$ To prepare compound $\mathbf{4}$, modified procedures are performed instead of the procedure in the previous literature. ${ }^{\mathrm{S} 2}$

Scheme S1. Synthetic scheme of compound 1.

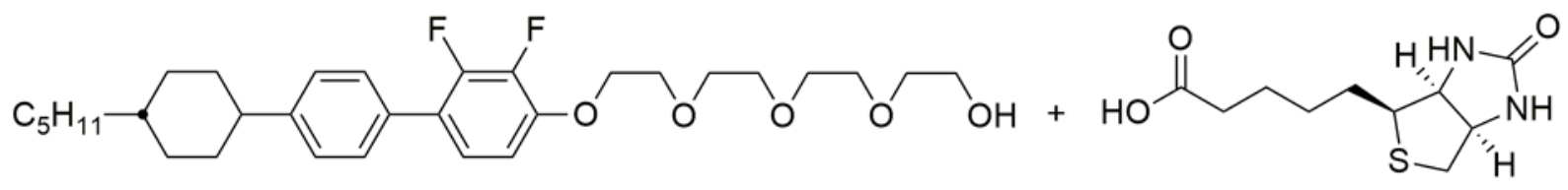

3

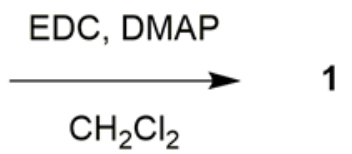


Scheme S2. Synthetic scheme of compound 2.
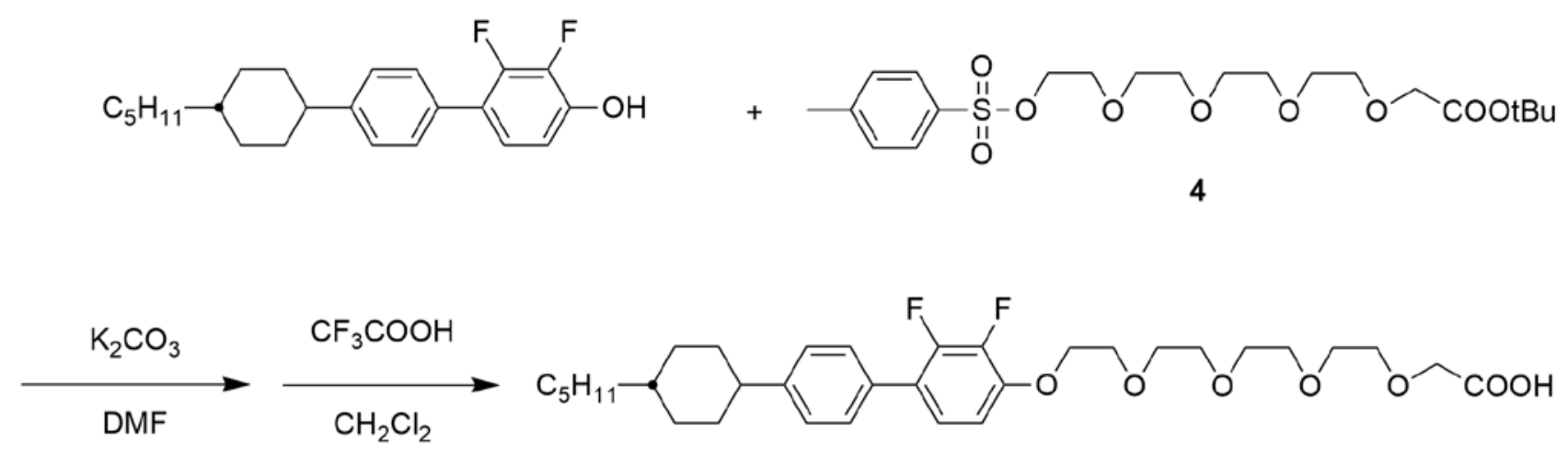

5
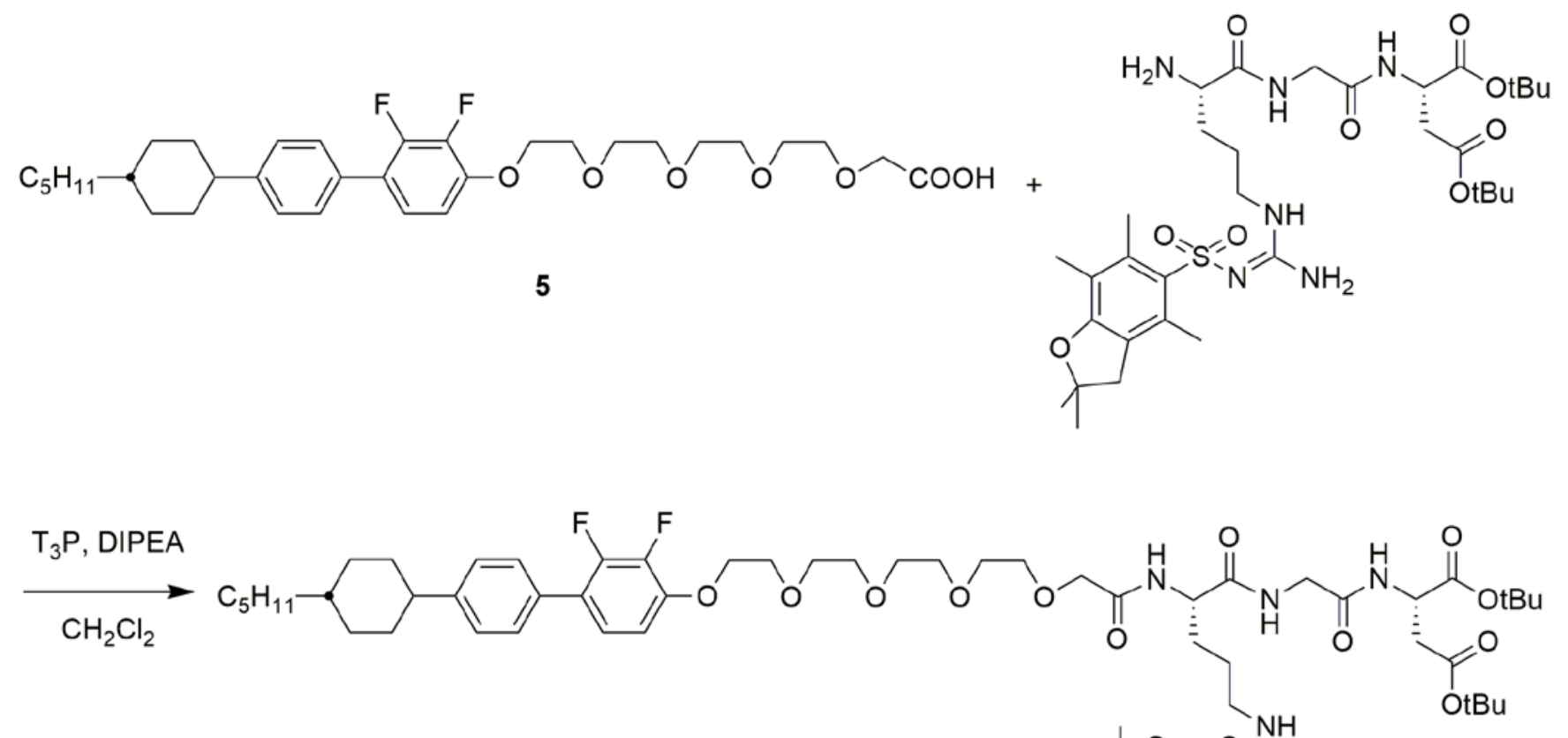

6<smiles>CNC(N)=NS(=O)(=O)c1c(C)c(C)c2c(c1C)CC(C)(C)O2</smiles>

$\mathrm{CF}_{3} \mathrm{COOH} /$ water/TIS

$(95: 2.5: 2.5)$ 
Hexahydro-2-oxo-1H-thieno[3,4-d] imidazole-4-pentanoic acid 2-(2-[2-\{2-(2,3-difluoro-4-\{4-(4-transpentylcyclohexyl)phenyl\}phenoxy)ethoxy\}ethoxy] ethoxy)ethanol ester (1).

A mixture of 3 (0.13 g, $0.24 \mathrm{mmol})$, biotin (0.059 g, $0.24 \mathrm{mmol})$, 1-ethyl-3-(3dimethylaminopropyl)carbodiimide hydrochloride (0.079 g, $0.41 \mathrm{mmol})$, 4-dimethylaminopyridine (0.0059 g, $0.048 \mathrm{mmol})$, and $\mathrm{CH}_{2} \mathrm{Cl}_{2}(5.0 \mathrm{~mL}$ ) was stirred at room temperature overnight. After adding $\mathrm{CH}_{2} \mathrm{Cl}_{2}$, the reaction mixture was washed with saturated $\mathrm{NH}_{4} \mathrm{Cl}$ aqueous solution, brine and then dried over $\mathrm{Na}_{2} \mathrm{SO}_{4}$. After filtration and evaporation, the crude material was purified by silica gel column chromatography $\left(\mathrm{CHCl}_{3} / \mathrm{MeOH}=19 / 1\right)$ and recrystallized from methanol to give $\mathbf{1}$ as white crystals. Yield: 0.041g (23\%: mp $\left.128{ }^{\circ} \mathrm{C}\right) .{ }^{1} \mathrm{H}$ NMR $\left(400 \mathrm{MHz}, \mathrm{CDCl}_{3}\right): \delta=7.42$ (d, J = $\left.6.8 \mathrm{~Hz}, 2 \mathrm{H}\right), 7.27$ (d, J $=8.0 \mathrm{~Hz}, 2 \mathrm{H}), 7.11-7.06(\mathrm{~m}, 1 \mathrm{H}), 6.84-6.80(\mathrm{~m}, 1 \mathrm{H}), 5.78(\mathrm{~s}, 1 \mathrm{H}), 5.16(\mathrm{~s}, 1 \mathrm{H}), 4.50-4.47(\mathrm{~m}, 1 \mathrm{H}), 4.31-$ 4.28 (m, 1H), 4.25-4.19 (m, 4H), $3.90(\mathrm{t}, \mathrm{J}=4.8 \mathrm{~Hz}, 2 \mathrm{H}), 3.76-3.66(\mathrm{~m}, 10 \mathrm{H}), 3.16-3.11(\mathrm{~m}, 1 \mathrm{H}), 2.90$ (dd, J = 12.8, $5.2 \mathrm{~Hz}, 1 \mathrm{H}$ ), 2.73 (d, J = 12.8 Hz, 1H), 2.53-2.47 (m, 1H), 2.37 (t, J = 7.6 Hz, 2H), 1.94$1.22(\mathrm{~m}, 21 \mathrm{H}), 1.10-1.02(\mathrm{~m}, 2 \mathrm{H}), 0.90$ (t, $7.2 \mathrm{~Hz}, 3 \mathrm{H}) .{ }^{13} \mathrm{C} \mathrm{NMR}\left(100 \mathrm{MHz}, \mathrm{CDCl}_{3}\right): \delta=173.67(\mathrm{~s})$, 163.39 (s), 148.88 (dd, 250, 11.5 Hz), 147.53 (s), 147.24 (d, 5.8 Hz), 141.88 (dd, 249, 14.9 Hz), 132.23 (s) 128.61 (d, 1.9 Hz), 127.07 (s), 123.58 (s), 123.52 (d, 9.6 Hz), 110.02 (d, 2.9 Hz),70.98 (s), 70.65 (s), 70.61 (s), 70.54 (s), 69.56 (s), 69.51 (s), 69.15 (s), 63.44 (s), 61.90 (s), 60.08 (s), 55.46 (s), 44.36 (s), 40.54 (s), 37.38 (s), 37.32 (s), 34.30 (s), 33.75 (s), 33.59 (s), 32.22 (s), 28.28 (s), 28.21 (s), 26.66 (s), 24.73 (s), 22.73 (s), 14.13 (s). IR (KBr): v 3252, 3078, 2922, 2852, 1731, 1697, 1650, 1507, 1471, 1412, 1387, 1351 1317, 1301, 1269, 1189, 1140, 1107, 1083, 956, 897, 871, 837, $799 \mathrm{~cm}^{-1}$. Anal. Calcd for $\mathrm{C}_{41} \mathrm{H}_{58} \mathrm{~F}_{2} \mathrm{~N}_{2} \mathrm{O}_{7} \mathrm{~S}$ (760.97): C, 64.71; H, 7.68; N, 3.68. Found: C, 64.47; H, 7.65; N, 3.72. MS (MALDITOF): calcd, $761.40\left([\mathrm{M}+\mathrm{H}]^{+}\right)$; found, 761.47 .

14-(Toluene-4-sulfonyloxy)-3,6,9,12-tetraoxatetradecanoic acid, t-butyl ester (4). 
To a solution of $t$-butyl 14-hydroxy-3,6,9,12-tetraoxatetradecanoate (2.2 g, $7.0 \mathrm{mmol})$, and triethylamine $(0.87 \mathrm{~g}, 8.6 \mathrm{mmol})$ in $\mathrm{CH}_{2} \mathrm{Cl}_{2}(10 \mathrm{ml})$ was added tosyl chloride $(1.3 \mathrm{~g}, 6.7 \mathrm{mmol})$ at $0{ }^{\circ} \mathrm{C}$, stirred at room temperature overnignt. After adding brine, the mixture was extracted three times with dichloromethane. The combined organic phase was dried over $\mathrm{MgSO}_{4}$ and the solvent was removed in vacuo. The residue was purified by silica gel column chromatography (from $\mathrm{CHCl}_{3} / \mathrm{AcOEt}=9 / 1$ to 4/1) to afford 4 as colorless liquid. Yield: 0.88g (28\%). ${ }^{1} \mathrm{H}$ NMR (400 MHz, $\left.\mathrm{CDCl}_{3}\right): \delta=7.80(\mathrm{~d}, \mathrm{~J}=8.4 \mathrm{~Hz}$, 2H), 7.34 (d, J = 8.4 Hz, 2H), 4.16 (t, J = 5.0 Hz, 2H), 4.01 (s, 2H), 3.72-3.58 (m, 14H), 2.45 (s, 3H), $1.47(\mathrm{~s}, 9 \mathrm{H})$

\section{2-\{2-(2-[2-\{2-(2,3-Difluoro-4-\{4-(4-trans-}

pentylcyclohexyl)phenyl\}phenoxy)ethoxy\}ethoxy]ethoxy)ethoxy\}acetic acid (5).

A mixture of 2,3-difluoro-4'-(4-pentylcyclohexyl)-4-biphenylol (0.69 g, 1.9 mmol), 4 (0.88 g, 1.9 $\mathrm{mmol}), \mathrm{K}_{2} \mathrm{CO}_{3}(0.80 \mathrm{~g}, 5.8 \mathrm{mmol})$ in dry $\mathrm{DMF}(5.0 \mathrm{~mL})$ was stirred at $80{ }^{\circ} \mathrm{C}$ for $6 \mathrm{~h}$. The reaction mixture was poured into ethyl acetate and washed with brine twice, dried over $\mathrm{Na}_{2} \mathrm{SO}_{4}$. After evaporation solvent, the residue was purified by silica gel column chromatography (hexane/ethyl acetate $=1 / 1$ ) to obtain $t$-butyl ester of 5 . Yield $1.0 \mathrm{~g}(84 \%)$. This compound was immediately used for the next reaction. ${ }^{1} \mathrm{H}$ NMR (400 MHz, $\left.\mathrm{CDCl}_{3}\right): \delta=7.42(\mathrm{~d}, \mathrm{~J}=6.8 \mathrm{~Hz}, 2 \mathrm{H}), 7.27(\mathrm{~d}, \mathrm{~J}=8.0 \mathrm{~Hz}, 2 \mathrm{H}), 7.11-7.06$ (m, 1H), 6.84-6.80 (m, 1H), 4.24 (t, $4.8 \mathrm{~Hz}, 2 \mathrm{H}), 4.02$ (s, 2H), 3.90 (t, J = 4.8 Hz, 2H), 3.76-3.67 (m, 12H), 2.53-2.46 (m, 1H), 1.94-1.86 (m, 4H), 1.55-1.19 (m, 20H), 1.11-1.01 (m, 2H), 0.90 (t, 7.2 Hz, 3H). t-Butyl ester of 5 (1.0 g, $1.6 \mathrm{mmol})$ was successively dissolved in $\mathrm{CH}_{2} \mathrm{Cl}_{2}(9.0 \mathrm{ml})$. After adding trifluoroacetic acid $(1.0 \mathrm{ml})$ at $0{ }^{\circ} \mathrm{C}$, the solution was stirred at room temperature overnight. After evaporation, the crude product was purified by silica gel column chromatography (ethyl acetate only and chloroform/ethyl acetate $=$ from $1 / 0$ to $1 / 1)$ to give 5 . Yield: 0.80 g (85\%). ${ }^{1} \mathrm{H}$ NMR (400 MHz, $\left.\mathrm{CDCl}_{3}\right)$ : 
$\delta=7.42(\mathrm{~d}, \mathrm{~J}=8.0 \mathrm{~Hz}, 2 \mathrm{H}), 7.27(\mathrm{~d}, \mathrm{~J}=8.0 \mathrm{~Hz}, 2 \mathrm{H}), 7.11-7.06(\mathrm{~m}, 1 \mathrm{H}), 6.84-6.80$ (m, $1 \mathrm{H}), 4.25$ (t, 4.8 Hz, 2H), 4.12 (s, 2H), 3.91 (t, J = 4.8 Hz, 2H), 3.79-3.67 (m, 12H), 2.54-2.47 (m, 1H), 1.94-1.87 (m, 4H), 1.53-1.43 (m, 2H), 1.33-1.22 (m, 9H), 1.11-1.01 (m, 2H), 0.90 (t, 7.2 Hz, 3H). ${ }^{13} \mathrm{C}$ NMR (100 MHz, $\mathrm{CDCl}_{3}$ ): $\delta=172.13$ (s), 148.82 (dd, 250, $10.6 \mathrm{~Hz}$ ), 147.50 (s), 147.20 (d, $5.7 \mathrm{~Hz}$ ), 141.88 (dd, $249,15.4$ Hz), 132.25 (s), 128.61 (d, $2.8 \mathrm{~Hz}), 127.06$ (s), 123.59 (s), 123.53 (d, $10.5 \mathrm{~Hz}$ ), 110.01 (s),71.44 (s), 71.03 (s), 70.68 (s), 70.47 (s), 70.35 (s), 70.21 (s), 69.52 (s), 69.39 (s), 69.03 (s), 44.36 (s), 37.39 (s), 37.32 (s), 34.30 (s), 33.60 (s), 32.23 (s), 26.66 (s), 22.73 (s), 14.13 (s). IR (KBr): v 3342, 3029, 2922, 2850, 1754, 1735, 1629, 1504, 1457, 1409, 1349, 1315, 1298, 1232, 1201, 1112, 1019, 958, 897, 795 $\mathrm{cm}^{-1}$. Anal. Calcd for $\mathrm{C}_{33} \mathrm{H}_{46} \mathrm{~F}_{2} \mathrm{O}_{7}$ (592.71): C, 66.87; H, 7.82. Found: C, 66.71; H, 7.79. MS (MALDITOF): calcd, $615.31\left([\mathrm{M}+\mathrm{Na}]^{+}\right)$; found, 615.08 .

$N^{\alpha}-[2-\{2-(2-[2-\{2-(2,3-D i f l u o r o-4-\{4-(4-t r a n s-$

pentylcyclohexyl)phenyl\}phenoxy)ethoxy\}ethoxy] ethoxy)ethoxy\}acetyl]- $N^{\omega}-(2,2,4,6,7-$

pentamethyldihydrobenzofuran-5-sulfonyl)-L-argnylglycyl-L-aspartic acid, $\alpha, \gamma$-bis(t-butyl) ester (6).

To a solution of 5 (0.29 g, $0.49 \mathrm{mmol})$, protected RGD motif (0.36 g, $0.50 \mathrm{mmol})$, and diisopropylethylamine $(0.14 \mathrm{~g}, 1.1 \mathrm{mmol})$ in dry $\mathrm{CH}_{2} \mathrm{Cl}_{2}(6.0 \mathrm{ml})$ was added $50 \mathrm{wt} \%$ propylphosphoric anhydride solution in ethyl acetate $(360 \mu \mathrm{l})$ at $0{ }^{\circ} \mathrm{C}$, stirred at room temperature for $24 \mathrm{~h}$. After evaporation of the reaction mixture, the residue was purified by silica gel chromatography $\left(\mathrm{CHCl}_{3} / \mathrm{MeOH}=\right.$ from 98/2 to 92/8). Yield: 0.46g (73\%). ${ }^{1} \mathrm{H}$ NMR $\left(400 \mathrm{MHz}, \mathrm{CDCl}_{3}\right): \delta=7.54(\mathrm{~d}, \mathrm{~J}=$ 8.4 Hz, 1H), 7.51-7.48 (m, 1H), 7.41 (d, J = 8.0 Hz, 2H), 7.27 (d, J = 8.0 Hz, 2H), 7.10-7.06 (m, 1H), 7.00 (d, J = 8.0 Hz, 1H), 6.82-6.78 (m, 1H), 6.26 (s, 2H), 6.07 (s, 1H), 4.66-4.55 (m, 2H), 4.21 (t, 4.8 Hz, 2H), 4.06-3.92 (m, 4H), 3.88 (t, J = 4.6 Hz, 2H), 3.75-3.63 (m, 12H), 3.29-3.21 (m, 2H), 2.93 (s, $2 \mathrm{H})$, 2.84 (dd, J = 17.2, 4.6 Hz, 1H), 2.69 (dd, J = 17.2, 4.6 Hz, 1H), 2.59 (s, 3H), 2.51-2.47 (m, 4H), 2.08 (s, 
3H), 1.99-1.72 (m, 6H), 1.56-1.22 (m, 37H), 1.10-1.02 (m, 2H), 0.90 (t, 7.2 Hz, 3H). ${ }^{13} \mathrm{C}$ NMR (100 MHz, $\mathrm{CDCl}_{3}$ ): $\delta=172.11$ (s), 170.56 (s), 170.15 (s), 169.65 (s), 168.92 (s), 158.60 (s), 156.45 (s), 148.72 (dd, 251, 12.5 Hz), 147.50 (s), 147.15 (d, 7.7 Hz), 141.90 (dd, 248, 14.4 Hz), 138.36 (s), 133.15 (s), 132.29 (s), 132.20 (s), 128.61 (s), 127.05 (s), 124.50 (s), 123.61 (s), 123.55 (d, $11.5 \mathrm{~Hz}), 117.38$ (s), 109.97 (s), 86.29 (s), 82.51 (s), 81.65 (s), 71.05 (s), 70.84 (s), 70.58 (s), 70.45 (s), 70.24 (s), 69.48 (s), 69.40 (s), 52.04 (s), 44.36 (s), 37.39 (s), 37.32 (s), 34.29 (s), 33.58 (s), 32.21 (s), 29.57 (s), 28.59 (s), 28.03 (s), 27.86 (s), 26.65 (s), 25.33 (s), 22.72 (s), 19.29 (s), 17.96 (s), 14.12 (s), 12.47 (s). IR (KBr): v 3437, 3353, 2924, 1733, 1664, 1550, 1504, 1472, 1369, 1298, 1254, 1152, 1108, 894, 847, 806, $670 \mathrm{~cm}^{-}$

1. Anal. Calcd for $\mathrm{C}_{66} \mathrm{H}_{98} \mathrm{~F}_{2} \mathrm{~N}_{6} \mathrm{O}_{15} \mathrm{~S}$ (1285.58): C, 61.66; H, 7.68; N, 6.54. Found: C, 61.47; H, 7.77; N, 6.51. MS (MALDI-TOF): calcd, 1291.69 ([M+Li $\left.]^{+}\right)$; found, 1291.36.

$N^{\alpha}-[2-\{2-(2-[2-\{2-(2,3-D i f l u o r o-4-\{4-(4-t r a n s-$

pentylcyclohexyl)phenyl\}phenoxy)ethoxy\}ethoxy]ethoxy)ethoxy\}acetyl]-L-arginylglycyl-L-aspartic acid, 2,2,2-trifluoroacetate (2).

A mixture of 6 ( $0.13 \mathrm{~g}, 0.10 \mathrm{mmol})$ in trifluoroacetic acid, water, and triisopropylsilane (2.0 ml, 95 : 2.5: 2.5) was stirred for $1.5 \mathrm{~h}$. After removal of solvent, the residue was purified by ODS column chromatography $\left(\mathrm{H}_{2} \mathrm{O} / \mathrm{MeOH}=15 / 85\right.$ containing $0.1 \%$ trifluoroacetic acid). The waxy solid was dissolved in a mixture of water water/t-BuOH and lyphilised to afford 2 as a fluffy. Yield: $0.088 \mathrm{~g}$ (83\%: mp $192{ }^{\circ} \mathrm{C}$ dec). ${ }^{1} \mathrm{H}$ NMR (400 MHz, DMSO): $\delta=8.44$ (t, J = 5.6 Hz, 1H), 8.13 (d, J = $\left.7.6 \mathrm{~Hz}, 1 \mathrm{H}\right)$, 8.11 (br, 1H), 7.68 (d, J = 7.6 Hz, 1H), 7.42 (d, J = 6.8 Hz, 2H), 7.32-7.11 (m, 8H), 4.43-4.35 (m, 2H), 4.25 (t, J = 4.4 Hz, 2H), 5.84-5.81 (d, J = $10 \mathrm{~Hz}, 2 \mathrm{H}), 5.38-5.36(\mathrm{~d}, \mathrm{~J}=7.6 \mathrm{~Hz}, 1 \mathrm{H}), 4.80$ (m, 1H), 4.69 (m, 1H), 4.43 (m, 1H), 3.93 (s, 2H), 3.93-3.78 (m, 3H), 3.68-3.51 (m, 13H), 3.09 (m, 2H), 2.57-2.50 (m, 3H), 1.84-1.81 (m, 5H), 1.61-1.41 (m, 5H), 1.32-1.20 (m, 9H), 1.08-1.00 (m, 2H), 0.87 (t, J = 6.8 Hz, 
3H). ${ }^{13}$ C NMR (100 MHz, DMSO): $\delta=172.63$ (s), 172.35 (s), 171.39 (s), 169.17 (s), 168.26 (s), 156.81 (s), 147.88 (dd, 247, 10.6 Hz), 147.09 (s), 146.93 (d, 9.8 Hz), 140.60 (dd, 246, 15.4 Hz), 131.60 (s), 128.46 (d, 0.9 Hz), 127.06 (s), 124.07 (s), 122.18 (d, 10.5 Hz), 70.27 (s), 69.95 (s), 69.78 (s), 69.72 (s), 69.56 (s), 68.92 (s), 68.72 (s), 51.50 (s), 48.66 (s), 43.54 (s), 41.70 (s), 40.45 (s), 37.03 (s), 36.87 (s), 36.64 (s), 33.74 (s), 33.06 (s), 31.64 (s), 29.74 (s), 26.05 (s), 24.78 (s), 22.15 (s), 13.97 (s). IR (KBr): v 3357, 2923, 2853, 1665, 1537, 1505, 1471, 1412, 1316, 1298, 1202, 1134, 1108, 1084, 952, 897, 836, 800, $721 \mathrm{~cm}^{-1}$. Anal. Calcd for $\mathrm{C}_{47} \mathrm{H}_{67} \mathrm{~F}_{5} \mathrm{~N}_{6} \mathrm{O}_{14}$ (1035.06): C, 54.54; H, 6.52; N, 8.12. Found: C, 54.77; $\mathrm{H}$, 6.54; N, 8.11. MS (MALDI-TOF): calcd, $921.48\left(\left[\mathrm{M}-\mathrm{CF}_{3} \mathrm{CO}_{2}\right]^{+}\right)$; found, 921.13. 
2. Stability of monolayer for 2 on air-aqueous interface

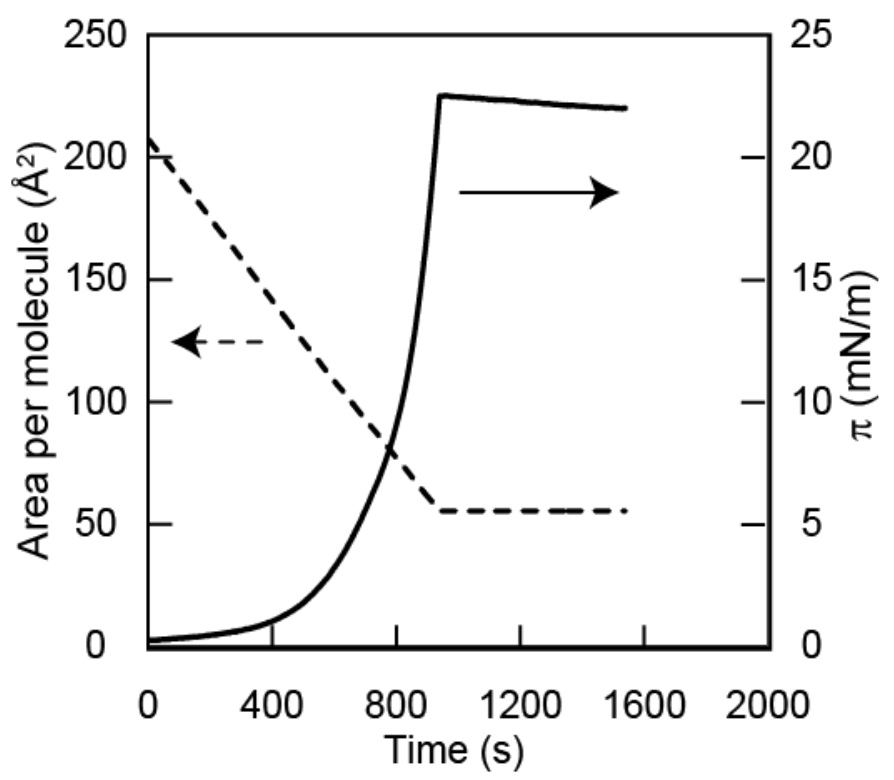

Figure S1. Change of area density and surface pressure for monolayers formed by compound 2 along time. Aqueous subphase is phosphate buffered saline (PBS) solution. 
3. Optical appearance under crossed polarizers of $5 \mathrm{CB} / 1$ and $5 \mathrm{CB} / 2$ between air and OTS-treated glass
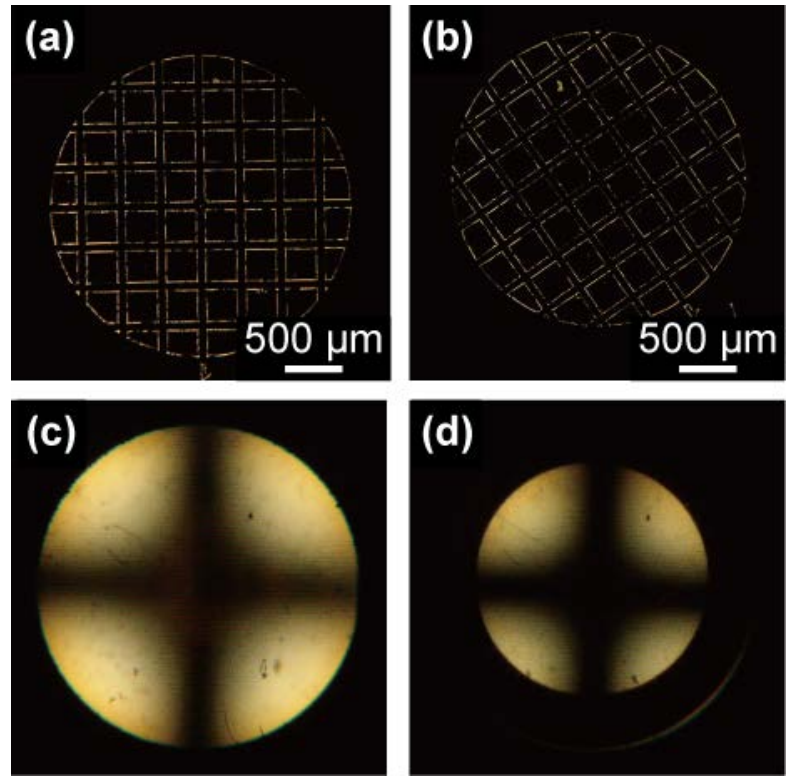

Figure S2. Optical micrographs (crossed polarizers) of optical liquid crystal cells for 5CB with 0.01 wt\% of 1 (a) and with 0.1 wt\% of $\mathbf{1}$ (b) in contact with air. Conoscopic images of optical liquid crystal cells for 5 CB with $0.01 \mathrm{wt} \%$ of $\mathbf{1}$ (c) and with $0.1 \mathrm{wt} \%$ of $\mathbf{1}$ (d) in contact with air.

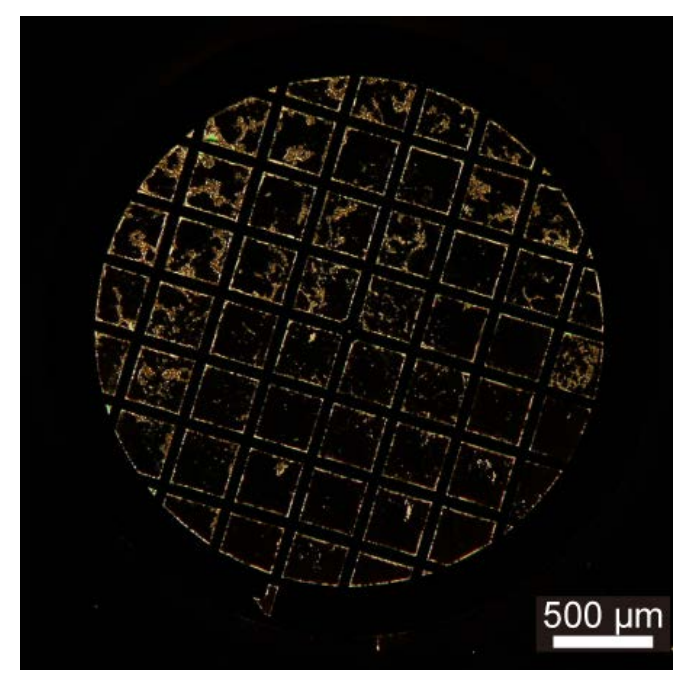

Figure S3. Optical micrograph (crossed polarizers) of optical liquid crystal cells for $\mathbf{5 C B}$ with $0.5 \mathrm{wt} \%$ of 2. 
4. Optical appearance under crossed polarizers of $5 \mathrm{CB} / 1$ between aqueous solution and OTStreated glass

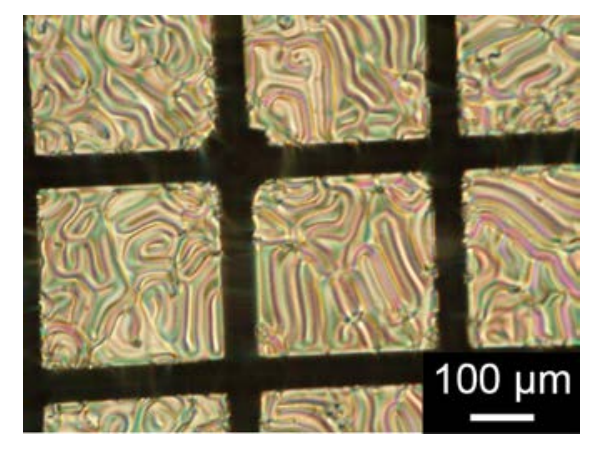

Figure S4. Optical micrograph (crossed polarizers) of optical liquid crystal cells for $\mathbf{5 C B}$ with 1 wt\% of $\mathbf{1}$ in contact with PBS solution.

\section{References}

(S1) Kishimoto, K.; Yoshio, M.; Mukai, T.; Yoshizawa, M.; Ohno, H.; Kato, T. J. Am. Chem. Soc. 2003, 125, 3196.

(S2) Van Boeckel, C. A. A.; Buijsman, R. C.; de Kort, M.; Meuleman, D. G. Eur. Pat. Appl. 1574516 A1 20050914, 2005.

(S3) Welsh, D. J.; Smith, D. K. Org. Biomol. Chem. 2011, 9, 4795. 